\title{
Scattering of surface waves by a semi-infinite floating elastic plate
}

\author{
T. Sahoo, T. L. Yip, and Allen T. Chwang ${ }^{\text {a) }}$ \\ Department of Mechanical Engineering, The University of Hong Kong, Pokfulam Road, Hong Kong
}

(Received 10 March 2000; accepted 17 July 2001)

\begin{abstract}
A new inner product is developed based on the Fourier analysis to study the scattering of surface waves by a floating semi-infinite elastic plate in a two-dimensional water domain of finite depth. The eigenfunctions for the plate-covered region are orthogonal with respect to this new inner product. The problem is studied for various wave and geometrical conditions. Especially, the influence of different edge conditions on the hydrodynamic behavior is investigated and compared. The edge conditions considered in the present study involve (i) a free edge, (ii) a simply supported edge, and (iii) a built-in edge. The hydrodynamic performance of an elastic plate is characterized for various conditions in terms of wave reflection and transmission, plate deflection, and surface strain. It is observed that the hydrodynamic behavior depends on the wave conditions, the geometrical settings, and the edge conditions. The built-in edge condition induces the maximum wave reflection and the minimum wave transmission. The free edge condition leads to the maximum plate deflection. () 2001 American Institute of Physics. [DOI: 10.1063/1.1408294]
\end{abstract}

\section{INTRODUCTION}

A great deal of effort is spent to effectively utilize the ocean space for human activities and developments. Certain huge platforms are constructed or extended from shoreline to provide more dry space, while infrastructures like floating ports, mobile offshore bases, are built as working spaces. At recent times, several artificial floating islands are constructed off shoreline. Such huge floating structures are categorized as Very Large Floating Structures (VLFS). Before the construction and positioning of any VLFS, careful and detailed studies are needed to investigate the hydrodynamic performance and hydroelastic behavior of the system. However, due to the large size of the structure, the computational burden becomes so large that it is often difficult to carry out the analysis. In order to overcome this difficulty, the structure is often assumed to be semi-infinitely long in comparison with the wavelength of the incident wave (Kagemoto et al. ${ }^{1}$ ). Under most considerations, marine bodies are assumed rigid in the presence of waves and their elastic deformations are neglected. However, the hydroelastic effect should be considered under certain wave conditions when (i) the body itself is flexible, (ii) the body is very thin compared to wave parameters, and (iii) the body is very long with respect to the incident wavelength. The former two cases should be quite obvious. However, in the latter case, localized deflection or vibration of a long structure becomes significant due to the continuous excitation of small amplitude waves, although the motion of the whole body is small as compared to its length.

In the theoretical study, there are two major approaches: The mode expansions method and the eigenfunction expansions method. In the mode expansions method, the body deformation is represented by a series of natural modes. The kinematic and dynamic surface conditions due to elasticity

${ }^{a)}$ Fax: (+852) 2858 5415. Electronic mail: atchwang@hkucc.hku.hk and gravity are treated separately. Based on the mode expansions method, Bishop and Price ${ }^{2}$ gave a comprehensive summary on the studies of hydroelasticity of ships, and $\mathrm{Gran}^{3}$ summed up the engineering applications of structural responses of marine structures to waves. Kashiwagi ${ }^{4}$ recently gave a review of the developments on VLFS and reported that major work on VLFS is based on the mode expansions method. It should be noted that the mode expansions method is only applicable to a finite plate. On the other hand, the eigenfunction expansions method is a more direct method, as it combines the kinematic and dynamic surface conditions, which give the dispersion relation satisfied by the wave numbers. Fox and Squire ${ }^{5}$ used the eigenfunction expansions method to study the interaction of surface waves with an ice-covered surface and obtained the solution by the conjugate gradient method. They observed that the eigenfunctions are not orthogonal with respect to the conventional inner product, though the eigenfunctions are complete. Squire et al. ${ }^{6}$ presented an invited review on the interaction of gravity waves with an ice-covered surface. Meylan ${ }^{7}$ derived a variational equation to deal with the wave response of an elastic plate of shallow draft. Meylan ${ }^{8}$ used Rayleigh-Ritz method to analyze the forced vibration of an arbitrary thin plate floating on the surface of an infinite liquid. Recently, Balmforth and Craster ${ }^{9}$ developed a method based on the Fourier transform and Wiener-Hopf technique to study the scattering of gravity waves incident on an ice-covered ocean and obtained asymptotic and approximate solutions to the problem. It may be noted that in the mathematical model, ice is considered as a thin elastic plate.

To analyze the response of a thin horizontal elastic plate floating in waves, Ohkusu and Nanba ${ }^{10}$ combined the kinematic and dynamic surface conditions to obtain the free surface condition on the plate-covered surface and then solved the problem by the boundary integral method. Sturova ${ }^{11}$ used Fox and Squire's 5 approach to study the oblique incidence of 
surface waves onto an elastic band. Kim and Ertekin ${ }^{12}$ constructed a complete set of orthogonal eigenfunctions satisfying the dispersion relations and then obtained the solution explicitly for predicting the hydroelastic behavior of a shallow-draft VLFS. Nanba and Ohkusu ${ }^{13}$ analyzed the elastic response in waves of a large floating platform of thin plate configuration in both shallow water and deep water. It may be noted that the free surface condition gives an important information regarding the wave numbers.

In all these studies related to an elastic plate floating on the water surface, the plate is assumed to have a free edge, which suggests that the shear force and the bending moment of the plate vanish at the edge. However, artificial structures are usually kept fixed or moored at the edge by ropes, anchors, tension cables, or piles. In such cases, the free edge condition should be replaced by the simply supported edge condition or the built-in edge condition as per the reality. It may be noted that for the simply supported edge condition, the deflection and the bending moment are assumed to vanish, whereas for the built-in edge condition, the deflection and the slope of deflection will vanish. In the mode expansions method, Newman ${ }^{14}$ proposed to employ different orthogonal polynomials to represent the corresponding mode expansions for different edge conditions, and claimed that the application of natural modal functions should be limited to the free edge condition only. Newman ${ }^{14}$ noted that it is very difficult to identify the fittest modal functions for various edge conditions. Wu et al..$^{15}$ extended Newman's ${ }^{14}$ idea to analyze the wave-induced responses of an elastic floating plate. There is still no criterion to detect the appropriate modal function for each edge condition.

In the present paper, we investigate the interaction of surface waves with a semi-infinite elastic plate floating on the free surface in finite water depth. The hydrodynamic behaviors due to three different types of edge conditions, namely (i) free edge, (ii) simply supported edge, and (iii) built-in edge, are analyzed. Using a newly defined inner product along with the method of matched eigenfunction expansions, a full solution is obtained. The edge conditions are directly incorporated while using the matching condition along with the orthogonality property. The defined inner product is a generalization of the well-known gravity wave inner product developed by Havelock, ${ }^{16}$ which was generalized by Rhodes-Robinson ${ }^{17,18}$ to deal with problems related to capillary gravity waves. Chakrabarti and Sahoo ${ }^{19}$ applied the mixed Fourier transform and the orthogonal inner product of Rhodes-Robinson ${ }^{17,18}$ to study the surface wave scattering and generation by permeable vertical barriers in both finite and infinite depths in the presence of surface tension. In case of capillary gravity waves, the solution depends on an edge condition, which is supposed to be prescribed at the solid-water interaction point. In the present study, the full solution depends on the prescribed edge conditions, which are obtained from the edge behavior of the elastic plate. The inner product is defined by inspecting the corresponding boundary value problem of infinite water depth. The beauty of the present method is that, it can deal with not only the three kinds of edge conditions but also a plate of finite or infinite geometry. It may be noted that Kim and Ertekin ${ }^{12}$

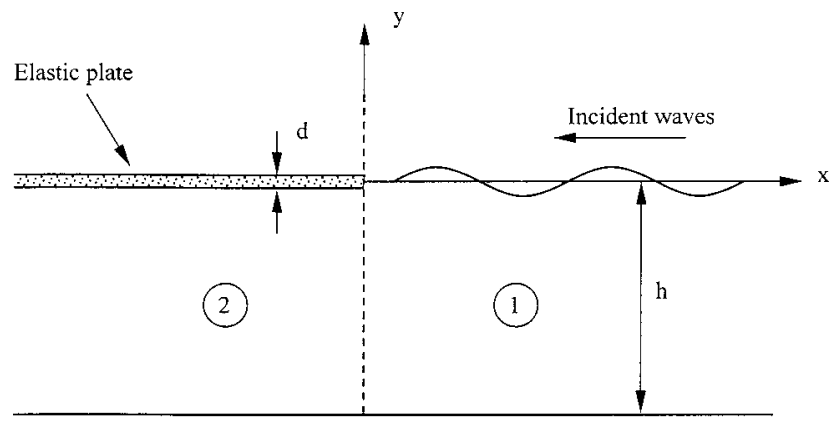

FIG. 1. Schematic diagram.

modified the eigenfunctions of Fox and Squire ${ }^{5}$ to make them orthogonal with respect to the standard inner product. While, in the present study we have used the same eigenfunctions of Fox and Squire ${ }^{5}$ and used a more general inner product such that the eigenfunctions are orthogonal with respect to the modified inner product.

\section{MATHEMATICAL FORMULATION}

The problem under consideration is two-dimensional in nature. A semi-infinite elastic plate of thickness $d$ floats on the surface $(-\infty<x<0, y=0)$ of a fluid which occupies the domain $(-\infty<x<\infty,-h<y<0)$ (see Fig. 1). Under the assumption that the fluid is inviscid, incompressible and the motion is irrotational, there exists a velocity potential $\Phi(x, y, t)$ which satisfies the Laplace equation

$$
\nabla^{2} \Phi=0 \quad \text { for } \quad-h<y<0, \quad-\infty<x<\infty,
$$

in the fluid region for all time $t$. The bottom boundary condition is given by

$$
\frac{\partial \Phi}{\partial y}=0, \quad \text { on } \quad y=-h, \quad-\infty<x<\infty .
$$

Under the assumption of a linearized theory of surface waves, the free surface condition in the absence of surface tension in the open water region is given by

$$
\frac{\partial^{2} \Phi}{\partial t^{2}}+g \frac{\partial \Phi}{\partial y}=0, \quad \text { on } y=0, \quad 0<x<\infty,
$$

where $g$ is the acceleration due to gravity.

Assuming that any particle which is once between the elastic plate and the water surface remains on it, the linearized kinematic and dynamic conditions in the absence of surface tension are given by

$$
\frac{\partial \eta}{\partial t}=\frac{\partial \Phi}{\partial y}, \quad \text { on } \quad y=0, \quad-\infty<x<0,
$$

and

$$
\frac{\partial \Phi}{\partial t}+\frac{P}{\rho}+g \eta=0, \quad \text { on } \quad y=0, \quad-\infty<x<0,
$$

where $\eta$ is the transverse displacement of the elastic plate, $\rho$ the fluid density, and $P$ the surface pressure. It is assumed that the plate is a thin homogeneous elastic material with 
uniform mass density $\rho_{s}$ and thickness $d$. With these assumptions, the displacement $\eta$ is related to the differential pressure $P_{s}$ by

$$
P_{s}=E I \frac{\partial^{4} \eta}{\partial x^{4}}+m_{s}\left(\frac{\partial^{2} \eta}{\partial t^{2}}+g\right)
$$

where $E I=E d^{3} /\left[12\left(1-\nu^{2}\right)\right]$ is the flexural rigidity of the plate, $E$ is the effective Young's modulus of the elastic plate, $\nu$ is Poisson's ratio, $m_{s}=\rho_{s} d$ is the density of the elastic plate. When the surface pressure $P$ in the linearized dynamic condition (5) is equated to the sum of $P_{s}$, we derive from (4) to (6), the condition for $\Phi(x, y, t)$ as

$$
\begin{aligned}
&\left(E I \frac{\partial^{4}}{\partial x^{4}}+m_{s} \frac{\partial^{2}}{\partial t^{2}}+\rho g\right) \frac{\partial \Phi}{\partial y}+\rho \frac{\partial^{2} \Phi}{\partial t^{2}}=0, \\
& \text { on } \quad y=0, \quad-\infty<x<0 .
\end{aligned}
$$

Further, it is assumed that at the edge, where the plate meets the water surface, one of the following edge conditions are satisfied at $(0-, 0)$ (Timoshenko and Woinowsky-Krieger, ${ }^{20}$ p. 83):

(i) Free edge. In this case, there is zero bending moment and zero shear force at the edge of the plate, which suggests that $\partial^{2} \eta / \partial n^{2}$ and $\partial^{3} \eta / \partial n^{3}$ are zero at $(0-, 0)$ where $n$ is the normal in the outward direction. Using condition (4) and eliminating $\eta$ we derive that for a free edge plate, the edge condition is

$$
\frac{\partial^{2}}{\partial x^{2}}\left(\frac{\partial \Phi}{\partial y}\right)=0, \quad \frac{\partial^{3}}{\partial x^{3}}\left(\frac{\partial \Phi}{\partial y}\right)=0, \quad \text { at } \quad(x, y)=(0,0) .
$$

(ii) Simply supported edge. At a simply supported edge the displacement is zero and there is no bending moment, which suggests that $\eta$ and $\partial^{2} \eta / \partial n^{2}$ are zero at $(0-, 0)$. Using condition (4) and eliminating $\eta$ we derive that for a simply supported edge plate, the edge condition is

$$
\frac{\partial^{2}}{\partial x^{2}}\left(\frac{\partial \Phi}{\partial y}\right)=0, \quad\left(\frac{\partial \Phi}{\partial y}\right)=0, \quad \text { at } \quad(x, y)=(0,0) .
$$

(iii) Built-in edge. At a built-in edge, the displacement and the slope are zero at the edge of the plate, which suggests that $\eta$ and $\partial \eta / \partial n$ are zero at $(0-, 0)$. Using condition (4) and eliminating $\eta$ we derive that for a built-in edge plate, the edge condition is

$$
\frac{\partial}{\partial x}\left(\frac{\partial \Phi}{\partial y}\right)=0, \quad\left(\frac{\partial \Phi}{\partial y}\right)=0, \quad \text { at } \quad(x, y)=(0,0) .
$$

As the velocity and pressure are continuous across the boundary between the plate-covered region and the open water region (see Fig. 1), the full solution is obtained by matching the velocity and pressure at the boundary which gives

$$
\begin{aligned}
& \frac{\partial \Phi(0-, y, t)}{\partial x}=\frac{\partial \Phi(0+, y, t)}{\partial x} \text { for }-h<y<0, \\
& \Phi(0-, y, t)=\Phi(0+, y, t), \quad \text { for }-h<y<0 .
\end{aligned}
$$

Under the assumption that the motion is simple harmonic in time with angular frequency $\omega$, we can rewrite $\Phi$ as $\Phi(x, y, t)=\operatorname{Re}\{\phi(x, y) \exp (-i \omega t)\}$. Then the spatial velocity potential $\phi(x, y)$ satisfies Eq. (1) along with boundary conditions (2), (8), and (9). From (3), the free surface boundary condition in the open water region is given by

$$
g \frac{\partial \phi}{\partial y}-\omega^{2} \phi=0, \quad \text { on } \quad y=0, \quad 0<x<\infty .
$$

The water surface boundary condition beneath the elastic plate (7) is rewritten as

$$
\begin{aligned}
& E I \frac{\partial^{4}}{\partial x^{4}}\left(\frac{\partial \phi}{\partial y}\right)+\left(\rho g-m_{s} \omega^{2}\right) \frac{\partial \phi}{\partial y}-\rho \omega^{2} \phi=0, \\
& \text { on } \quad y=0, \quad-\infty<x<0 .
\end{aligned}
$$

\section{METHOD OF SOLUTION}

The fluid domain is divided into two regions: The upstream open region $(x>0,-h<y<0)$ and the plate-covered region $(x<0,-h<y<0)$. The spatial velocity potentials in the corresponding regions are expressed as

$$
\begin{aligned}
\phi_{1}= & \left(I_{0} e^{-i k_{0} x}+R_{0} e^{i k_{0} x}\right) \psi_{0} \\
& +\sum_{m=1}^{\infty} R_{m} e^{-k_{m} x} \psi_{m}, \quad \text { for } x>0, \\
\phi_{2}= & T_{0} e^{-i p_{0} x} f_{0}+\sum_{n=I}^{I V} T_{n} e^{p_{n} x} f_{n} \\
& +\sum_{n=1}^{\infty} T_{n} e^{p_{n} x} f_{n}, \quad \text { for } \quad x<0,
\end{aligned}
$$

where

$$
\begin{aligned}
& \phi= \begin{cases}\phi_{1} & (x>0) \\
\phi_{2} & (x<0)\end{cases} \\
& \psi_{m}=\left\{\begin{array}{ll}
\frac{\cosh k_{0}(h+y)}{\cosh k_{0} h} & (m=0) \\
\frac{\cos k_{m}(h+y)}{\cos k_{m} h} & (m=1,2,3, \ldots)
\end{array},\right. \\
& f_{n}=\left\{\begin{array}{ll}
\frac{\cosh p_{0}(h+y)}{\cosh p_{0} h} & (n=0) \\
\frac{\cos p_{n}(h+y)}{\cos p_{n} h} & (n=\mathrm{I}, \mathrm{II}, \mathrm{III}, \mathrm{IV}, 1,2,3, \ldots)
\end{array},\right. \\
& I_{0}=-\frac{i g H_{0}}{2 \omega},
\end{aligned}
$$

with $H_{0}$ being the incident wave height. The constants $k_{n}$ 's satisfy the dispersion relations

$\omega^{2}=g k_{0} \tanh k_{0} h=-g k_{n} \tan k_{n} h \quad(n=1,2,3, \ldots)$, 
with $\left[(n-1) \pi / h<k_{n}<n \pi / h\right](n=1,2,3, \ldots)$. The eigenfunctions $\psi_{m}(m=0,1,2, \ldots)$ are orthogonal and complete in the usual sense. On the other hand, $p_{n}$ 's satisfy the dispersion relations

$$
\begin{aligned}
K & =p_{0}\left(1+L p_{0}^{4}\right) \tanh p_{0} h \\
& =-p_{n}\left(1+L p_{n}^{4}\right) \tan p_{n} h \quad(n=\mathrm{I}, \mathrm{II}, \mathrm{III}, \mathrm{IV}, 1,2,3, \ldots),
\end{aligned}
$$

with $L=E I /\left(\rho g-m_{s} \omega^{2}\right)$ and $K=\rho \omega^{2} /\left(\rho g-m_{s} \omega^{2}\right)$. It should be noted that $p_{\mathrm{I}}$ and $p_{\mathrm{II}}$ are complex conjugates with positive real parts, $p_{\mathrm{III}}$ and $p_{\mathrm{IV}}$ are complex conjugates with negative real parts, $p_{n}$ 's are positive and real $[(n-1) \pi /$ $\left.h<p_{n}<n \pi / h\right](n=1,2,3, \ldots)$, and $R_{n}, T_{n}(n=0, \mathrm{I}, \mathrm{II}, \mathrm{III}, \mathrm{IV}$, $1,2,3, \ldots)$ are unknown constants to be determined to obtain the velocity potentials completely. The assumption that the velocity potentials are bounded at infinity suggests that

$$
T_{\mathrm{III}}=T_{\mathrm{IV}}=0 \text {. }
$$

Define the inner products as

$$
\begin{aligned}
\left\langle f_{m}, f_{n}\right\rangle= & \int_{-h}^{0} f_{m} f_{n} d y+\frac{L}{K}\left\{\frac{\partial f_{m}}{\partial y} \frac{\partial^{3} f_{n}}{\partial y^{3}}+\frac{\partial^{3} f_{m}}{\partial y^{3}} \frac{\partial f_{n}}{\partial y}\right\}_{y=0} \\
& (m, n=0, \text { I,II,III,IV }, 1,2,3, \ldots) .
\end{aligned}
$$

Then, it is easy to derive that

$\left\langle f_{m}, f_{n}\right\rangle=0, \quad$ for $\quad m \neq n(m, n=0, \mathrm{I}, \mathrm{II}, \mathrm{III}, \mathrm{IV}, 1,2,3, \ldots)$,

$$
\left\langle f_{0}, f_{0}\right\rangle=\frac{2 p_{0} h\left(1+L p_{0}^{4}\right)+\left(1+5 L p_{0}^{4}\right) \sinh 2 p_{0} h}{4 p_{0}\left(1+L p_{0}^{4}\right)},
$$

$$
\left\langle f_{n}, f_{n}\right\rangle=\frac{2 p_{n} h\left(1+L p_{n}^{4}\right)+\left(1+5 L p_{n}^{4}\right) \sin 2 p_{n} h}{4 p_{n}\left(1+L p_{n}^{4}\right)}
$$

$$
(n=\mathrm{I}, \mathrm{II}, \mathrm{III}, \mathrm{IV}, 1,2,3, \ldots) \text {, }
$$

which suggests that the set of functions $\left\{f_{n} ; n\right.$ $=0, \mathrm{I}, \mathrm{II}, \mathrm{III}, \mathrm{IV}, 1,2, \ldots\}$ are orthogonal with respect to the inner product as defined by relation (17). However, the eigenfunctions in the plate-covered region are not standard ones as the operator involved is not self-adjoint. In the present analysis we assume that the above sequence of functions $\left\{f_{n}\right\}$ form a complete set for functions satisfying the same boundary value problems (e.g., Fox and Squire ${ }^{5}$ ). The detailed proof of completeness needs special attention as a new class of problems and is not of concern in the present context.

Using the inner product defined in (17) and the continuity of velocity (9a), we have

$$
\begin{aligned}
\left\langle\phi_{2 x}, f_{n}\right\rangle= & \int_{-h}^{0} \phi_{2 x} f_{n} d y+\frac{L}{K}\left\{\phi_{2 x y}(0,0) f_{n}^{\prime \prime \prime}(0)\right. \\
& \left.+\phi_{2 x y y y}(0,0) f_{n}^{\prime}(0)\right\} \\
= & \int_{-h}^{0} \phi_{1 x} f_{n} d y+\frac{L}{K}\left\{\phi_{2 x y}(0,0) f_{n}^{\prime \prime \prime}(0)\right. \\
& \left.+\phi_{2 x y y y}(0,0) f_{n}^{\prime}(0)\right\} \quad(n=0, \mathrm{I}, \mathrm{II}, 1,2,3, \ldots) .
\end{aligned}
$$

From (21), (12a) and (12b), we obtain

$$
\begin{aligned}
-i p_{0} T_{0}\left\langle f_{0}, f_{0}\right\rangle & \\
= & i k_{0}\left(R_{0}-I_{0}\right) \int_{-h}^{0} \psi_{0} f_{0} d y-\sum_{m=1}^{\infty} k_{m} R_{m} \int_{-h}^{0} \psi_{m} f_{0} d y \\
& +\frac{L}{K}\left[\phi_{2 x y}(0,0) f_{0}^{\prime \prime \prime}(0)+\phi_{2 x y y y}(0,0) f_{0}^{\prime}(0)\right], \\
p_{n} T_{n}\left\langle f_{n}, f_{n}\right\rangle & \\
= & i k_{0}\left(R_{0}-I_{0}\right) \int_{-h}^{0} \psi_{0} f_{n} d y-\sum_{m=1}^{\infty} k_{m} R_{m} \int_{-h}^{0} \psi_{m} f_{n} d y \\
& +\frac{L}{K}\left[\phi_{2 x y}(0,0) f_{n}^{\prime \prime \prime}(0)+\phi_{2 x y y y}(0,0) f_{n}^{\prime}(0)\right] \\
& (n=\mathrm{I}, \mathrm{II}, 1,2,3, \ldots) .
\end{aligned}
$$

Similarly, from Eqs. (9b) and (17), we have

$$
\begin{aligned}
T_{n}\left\langle f_{n}, f_{n}\right\rangle= & \left(R_{0}+I_{0}\right) \int_{-h}^{0} \psi_{0} f_{n} d y+\sum_{m=1}^{\infty} R_{m} \int_{-h}^{0} \psi_{m} f_{n} d y \\
& +\frac{L}{K}\left[\phi_{2 y}(0,0) f_{n}^{\prime \prime \prime}(0)+\phi_{2 y y y}(0,0) f_{n}^{\prime}(0)\right] \\
& (n=0, \mathrm{I}, \mathrm{II}, 1,2,3, \ldots) .
\end{aligned}
$$

Truncating the systems of Eqs. (22)-(24) after $N$ terms, we obtain a system of $(2 N+6)$ equations for $(2 N+6)$ unknowns $\quad R_{m} \quad$ and $T_{n} \quad(m=0,1,2, \ldots, N, N+1, N+2 ; n$ $=0, \mathrm{I}, \mathrm{II}, 1,2,3, \ldots, N)$. It may be noted that the edge conditions are applied based on the relation that $\partial^{2} \phi_{2} / \partial x^{2}$ $=-\partial^{2} \phi_{2} / \partial y^{2}$. The solutions in the three different cases are obtained by using the appropriate edge conditions.

\section{DISCUSSION ON NUMERICAL RESULTS}

The interaction of surface waves with a semi-infinite elastic plate is characterized by the reflection and transmission coefficients defined as

$$
K_{r}=\left|\frac{R_{0}}{I_{0}}\right|, \quad K_{t}=\mid \frac{p_{0} T_{0} \tanh p_{0} h}{k_{0} I_{0} \tanh k_{0} h} .
$$

The three dimensionless parameters are $k_{0} h, d / h$ and $E I / \rho g h^{4}$, while $H_{0} / h=0.01, \rho_{s} / \rho=1$ are kept throughout the computation. Because parameters like the density of water $\rho$, density of the plate $\rho_{s}$, and Poisson's ratio $\nu$ are limited to a small range of possible physical values, changing those parameters has negligible effect on wave profiles.

When an incident wave encounters a horizontal elastic plate floating on the free surface, part of the wave energy is reflected back to the upstream open region, while another part of the energy is transmitted into the plate-covered region. Under the elastic plate, there are two restoring forces acting on water, the elastic force due to the bending of the plate and the gravitational force. As a result, there are three kinds of waves acting below the plate: (i) an undamped progressive wave, (ii) evanescent wave modes, and (iii) a decaying progressive wave $\left(\right.$ Wadhams $^{21}$ ). The undamped progressive wave is associated with eigenvalue $p_{0}$ in (15). This wave is due to the flexural vibration of the plate and the 
gravity effect on waves in the covered region, which is called the flexural gravity wave. The evanescent wave modes exist because of the change of flow field. These evanescent wave modes are associated with eigenvalues $p_{n}(n=1,2,3, \ldots)$ in (15) with monotonic, exponentially decaying amplitudes. The decaying progressive wave is associated with eigenvalues $p_{\mathrm{I}}$ and $p_{\mathrm{II}}$ in (15). This wave can be better explained for the deep water case with simple algebra. Let $p_{\mathrm{I}}=a+i b$ and $p_{\mathrm{II}}=a-i b$, where $a$ and $b$ are positive real numbers. From (12b) and (13c), the spatial velocity potential associated with $p_{\mathrm{I}}$ and $p_{\mathrm{II}}$ for deep water $(h \rightarrow \infty)$ is

$$
\begin{aligned}
& T_{\mathrm{I}} e^{(a+i b) x} e^{(b-i a) y}+T_{\mathrm{II}} e^{(a-i b) x} e^{(b+i a) y} \\
& \quad=\left[2 T_{\mathrm{I}} \cos (b x-a y)+\left(T_{\mathrm{II}}-T_{\mathrm{I}}\right) e^{-i(b x-a y)}\right] e^{(a x+b y)}
\end{aligned}
$$

The first term in (26) is a standing wave or an evanescent wave with an oscillatory, exponentially decaying amplitude. The second term is a decaying progressive wave with a propagation vector $k=(-b, a)$, since the velocity potential is $\Phi=\operatorname{Re}\{\phi(x, y) \exp (-i \omega t)\}$. Physically, this represents a decaying progressive wave that propagates diagonally upwards and transfers energy from the open region/platecovered region boundary plane $(x=0)$ to the floating elastic plate as increased kinetic energy near the plate edge (Wadhams, ${ }^{21}$ p. 886).

\section{A. Loci of roots}

Figure 2 shows the loci of dimensionless wave number $p_{\mathrm{I}} h$ in a complex domain with varying $k_{0} h$ and $E I / \rho g h^{4}$. From the expression $\exp [(\alpha+i \beta) x]=\exp [\alpha x](\cos \beta x$ $+i \sin \beta x)(x<0)$, where $p_{\mathrm{I}}=\alpha+i \beta$, it is clear that the real part of $p_{\mathrm{I}} h$ denotes the decaying factor while the imaginary part of $p_{\mathrm{I}} h$ represents the wave number of progressive waves. Far away from the edge of the plate, the decaying progressive wave becomes negligible. When $k_{0} h$ increases, the real part of $p_{\mathrm{I}} h$ increases, and its imaginary part decreases generally [Fig. 2(a)]. For large $k_{0} h$ (or short incident waves), the decaying progressive wave becomes short (small $\beta$ ) and decays fast (large $\alpha$ ). As $E I / \rho g h^{4}$ increases, both real and imaginary parts of $p_{\mathrm{I}} h$ decreases generally [Fig. 2(b)]. Therefore, the decaying progressive wave propagates further (small $\alpha$ ) as the plate becomes more rigid.

\section{B. Reflection and transmission}

In Fig. 3 the variation of reflection coefficient $K_{r}$ and transmission coefficient $K_{t}$ versus $k_{0} h$ is plotted for various values of $E I / \rho g h^{4}$ for a free-edge plate. For long waves corresponding to a small wave number $k_{0} \mathrm{~h}$, the flow is uniform along the horizontal direction. As a result, there is little wave reflection by the horizontal plate for long waves. The plate appears to be transparent to the incident wave and the wave reflection disappears. In such a case, $K_{r}$ tends to zero and $K_{t}$ approaches one as $k_{0} h$ vanishes, which means that a major part of the wave energy for long waves is transmitted into the covered region. On the other hand, for large wave numbers, $K_{r}$ increases and $K_{t}$ decreases to zero. This is due to the fact that for short waves, which correspond to large

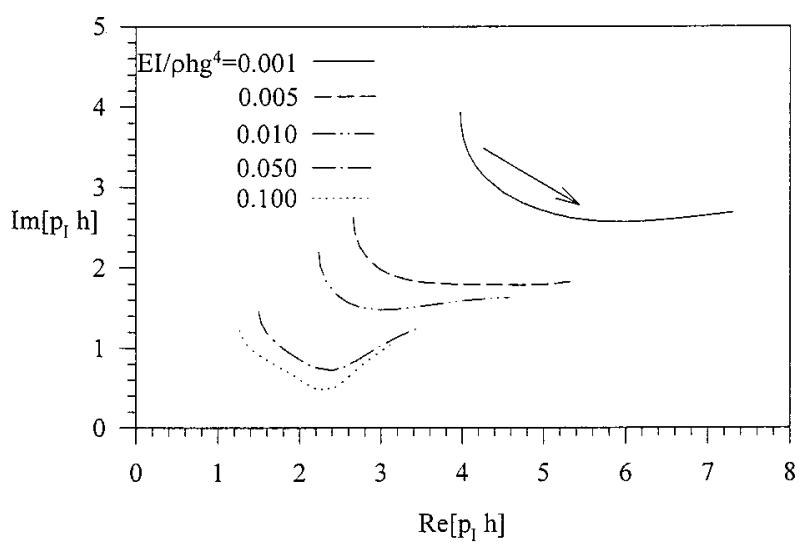

(a) $k_{0} h$ varies from 0.4 to 30.0

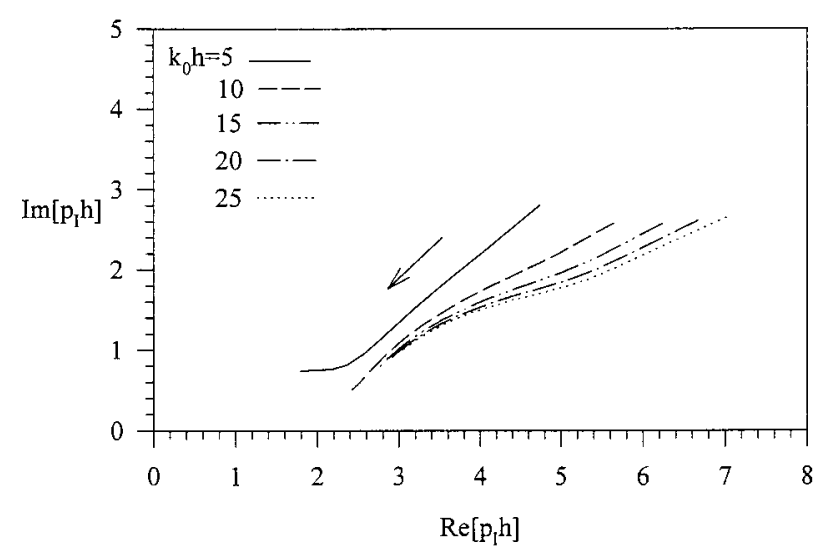

(b) El/pgh ${ }^{4}$ varies from 0.001 to 0.100

FIG. 2. Loci of $p_{\mathrm{I}} \mathrm{h}$ with $\mathrm{d} / \mathrm{h}=0.01$.

wave numbers, most of the wave energy is concentrated near the free surface. Thus, a large proportion of the wave energy is reflected back by the plate and a small amount of wave energy is transmitted. The rapid change of coefficients $K_{r}$ and $K_{t}$ is observed for moderate $k_{0} h$. Generally $K_{r}$ increases and $K_{t}$ decreases as $k_{0} h$ increases. Thus, incident waves of longer wavelength penetrate farther into the plate-covered region. For different $E I / \rho g h^{4}$, each curve of $K_{r}$ and $K_{t}$ has the same basic structure as $E I / \rho g h^{4}$ varies. As $E I / \rho g h^{4}$ in-

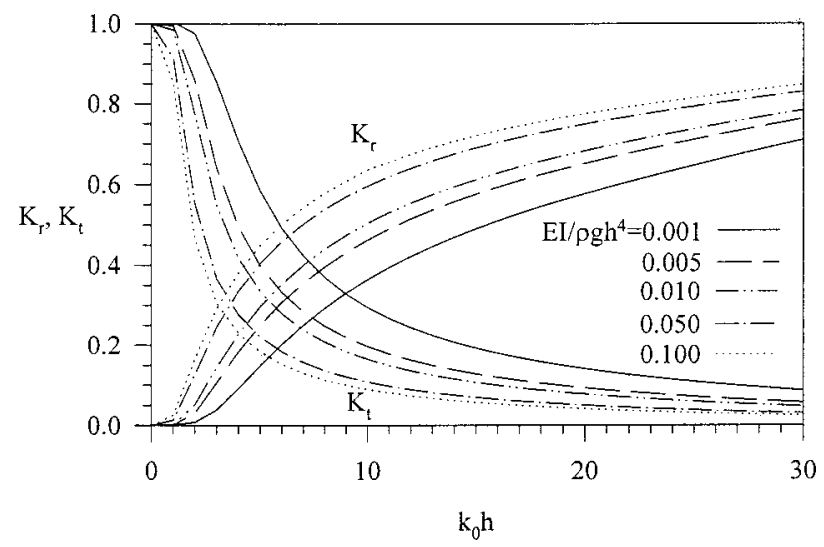

FIG. 3. Variation of $K_{r}$ and $K_{t}$ vs dimensionless wave number $k_{0} h$ for different values of $E I / \rho g h^{4}$ for a free-edge plate with $\mathrm{d} / \mathrm{h}=0.01$. 


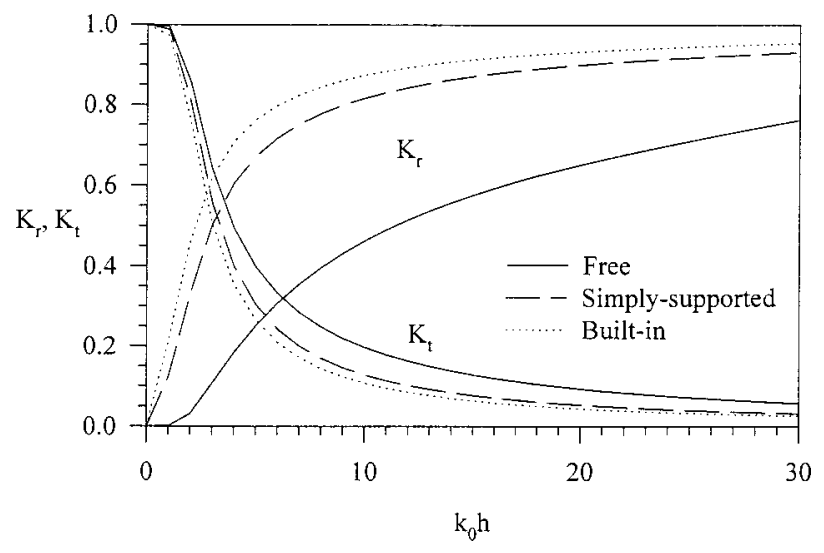

FIG. 4. Variation of $K_{r}$ and $K_{t}$ vs dimensionless wave number $k_{0} h$ for different edge conditions with $\mathrm{d} / \mathrm{h}=0.01$ and $E I / \rho g h^{4}=0.005$.

creases, the plate becomes rigid and most of the waves which concentrate near the free surface are reflected back and in the process less wave energy is transmitted below the plate.

Figure 4 illustrates the effect of different edge conditions, namely, free edge, simply supported edge and built-in edge on the scattering of surface waves. The reflection coefficient $K_{r}$ and transmission coefficient $K_{t}$ are plotted versus the dimensionless wave number for different types of edge conditions. It is observed that a free-edge plate exhibits lower $K_{r}$ and higher $K_{t}$, which indicates that a free-edge plate allows more incident wave energy to transmit below the plate and in turn the reflection becomes less. For a natural cover like ice, the wave transmission below the icecovered region helps in breaking ice in cold countries. In such a case, reflection is less compared to the plates having a built-in edge or a simply-supported edge. On the other hand, as $k_{0} h$ increases, the variations among wave reflection by the three types of edge condition diminish. The edge conditions are not only localized effects but also affect the wave scattering.

In Fig. 5, the variation of reflection and transmission coefficients is plotted versus $k_{0} h$ for various values of $N$ (number of terms in the evanescent wave modes for both $\phi_{1}$ and $\phi_{2}$ ). As $N$ increases beyond 20, no significant change in the reflection and transmission coefficients takes place as the

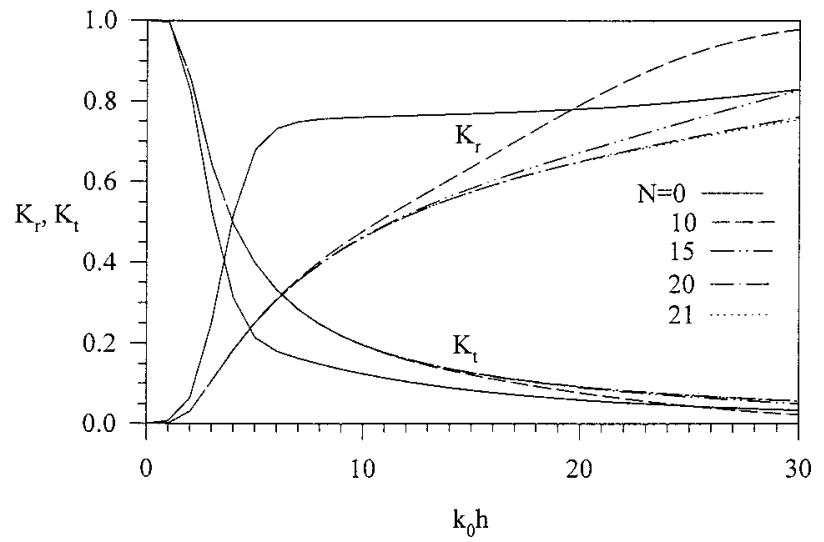

FIG. 5. Variation of $K_{r}$ and $K_{t}$ vs dimensionless wave number $k_{0} h$ for different number of terms $N$ with $\mathrm{d} / \mathrm{h}=0.01$ and $E I / \rho g h^{4}=0.005$.

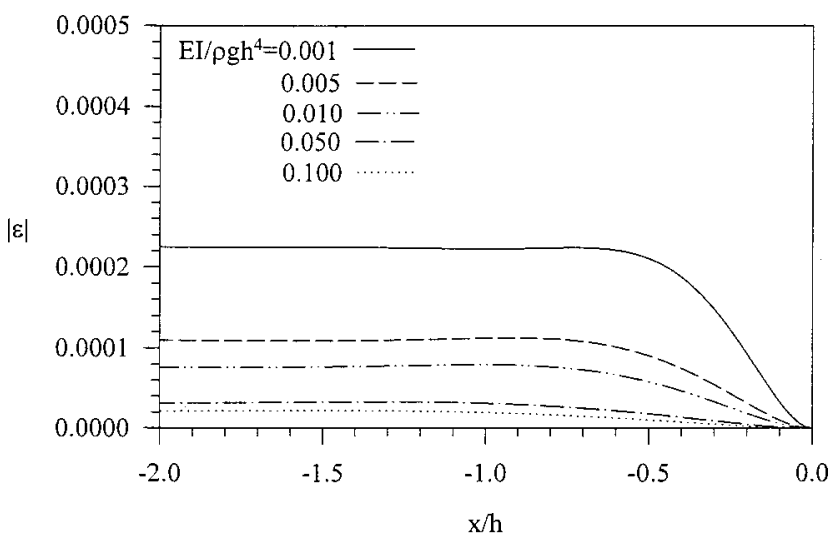

FIG. 6. Variation of strain $|\varepsilon|$ versus $x / h$ for different values of $E I / \rho g h^{4}$ for a free-edge plate with $\mathrm{d} / \mathrm{h}=0.01$ and $k_{0} h=4$.

higher order terms do not contribute much to the solution which is similar to that of Kim and Ertekin. ${ }^{12}$ The present result and the result of Kim and Ertekin ${ }^{12}$ are in sharp contrast to the observations of Fox and Squire. ${ }^{22}$ The slow convergence may be due to the wrong choice of Lagrange multipliers in the conjugate gradient method. Fox and Squire ${ }^{5}$ generalized the work of Fox and Squire ${ }^{22}$ and reported a more sophisticated scheme which operates in a subspace satisfying certain necessary conditions. More thought was also given to deep water. However, Fox and Squire ${ }^{5}$ have not mentioned anything regarding the number of evanescent modes used in their manuscript.

\section{Deflection and strain}

The deflection of the plate $\eta$ and the strain $\varepsilon$ at the plate surface are given by

$$
\begin{aligned}
& \eta=\left.i \frac{1}{\omega} \frac{\partial \phi}{\partial y}\right|_{y=0}, \\
& \varepsilon=\frac{d}{2} \frac{\partial^{2} \eta}{\partial x^{2}}=\left.i \frac{d}{2 \omega} \frac{\partial^{3} \phi}{\partial x^{2} \partial y}\right|_{y=0} .
\end{aligned}
$$

Figure 6 shows the variation of strain $|\varepsilon|$ versus $x / h$ for different values of $E I / \rho g h^{4}$ for a free-edge plate. It is ob-

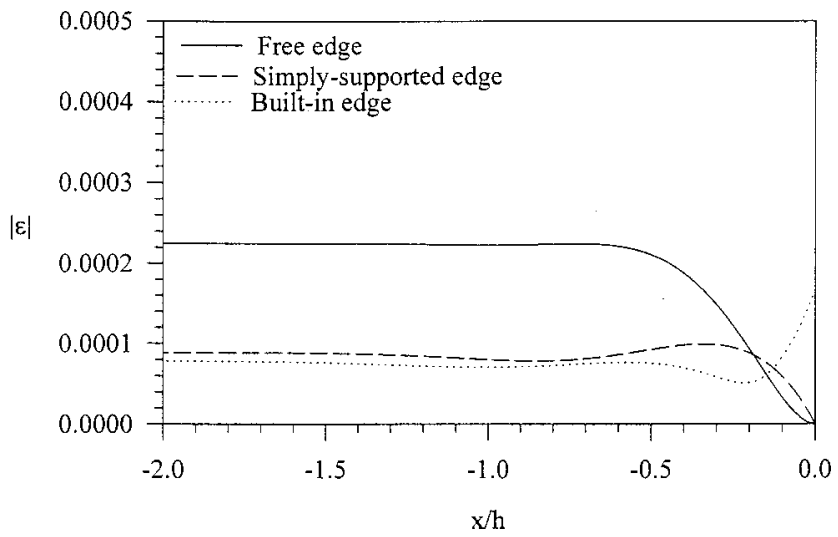

FIG. 7. Variation of strain $|\varepsilon|$ vs $x / h$ for different edge conditions with $\mathrm{d} / \mathrm{h}=0.01, k_{0} h=4$, and $E I / \rho g h^{4}=0.005$. 


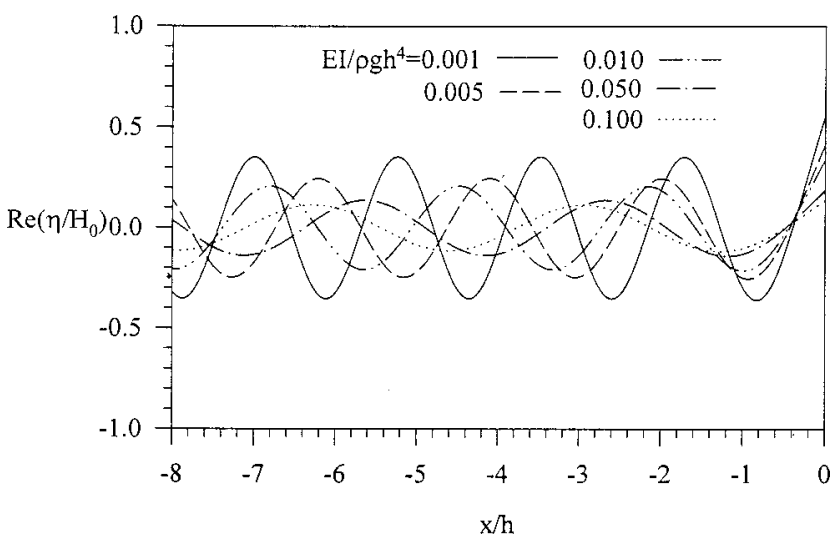

FIG. 8. Variation of dimensionless plate deflection $\operatorname{Re}\left(\eta / H_{0}\right)$ for different values of $E I / \rho g h^{4}$ for a free-edge plate with d/h=0.01 and $k_{0} h=4$.

served that strain $|\varepsilon|$ and its gradient vanish at the edge as the bending moment and shear force vanish there as prescribed. The strain on the plate highly depends on the flexural rigidity $E I / \rho g h^{4}$. Near the free edge, the plate excitation is very high for a flexible plate, which attains a steady state as the plate length increases. With the increase of plate length, the evanescent wave modes vanish and the plate oscillates due to the progressive wave. On the other hand, as EI/ $\rho g h^{4}$ increases, the plate becomes more rigid and thus the strain decreases.

Figure 7 shows the variation of surface strain $|\varepsilon|$ versus $x / h$ for different edge conditions. For a plate having a free edge or a simply supported edge, the strain vanishes at the edge, as the moment is prescribed to be zero there, while the plate with a built-in edge has finite nonzero strain near the edge. However, plates having different edge conditions show similar surface strain behavior away from the edge. As discussed before, the transmission coefficient is maximum for a free-edge plate. As more wave energy is transmitted below the plate, it excites the plate deflection regardless of edge conditions. On the other hand, it has a serious structural effect as the strain is maximum for a free-edge plate. For a free-edge plate, the deflection of the plate remains high near the edge because the decaying progressive wave and evanescent wave modes play a significant role there. Such behavior

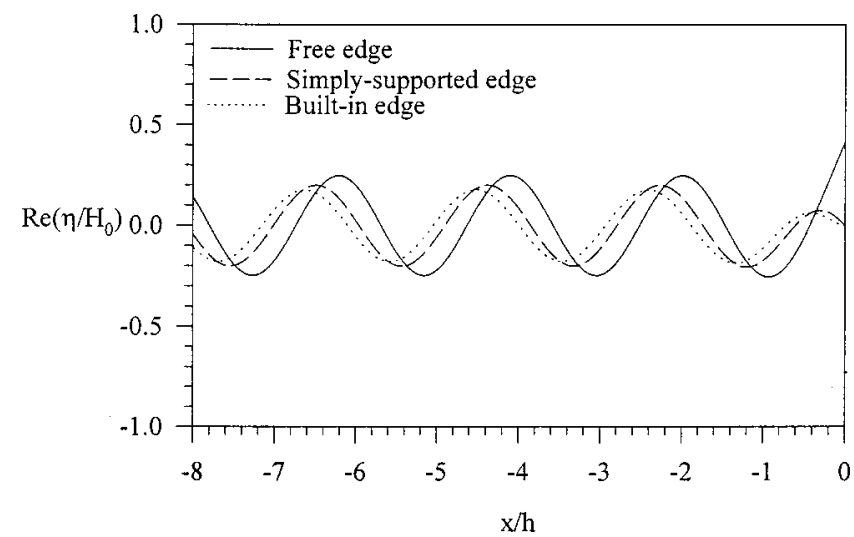

FIG. 9. Variation of dimensionless plate deflection $\operatorname{Re}\left(\eta / H_{0}\right)$ for different edge conditions with $E I / \rho g h^{4}=0.005, \mathrm{~d} / \mathrm{h}=0.01$, and $k_{0} h=4$.



FIG. 10. Variation of dimensionless plate deflection $\operatorname{Im}\left(\eta / H_{0}\right)$ for different edge conditions with $E I / \rho g h^{4}=0.005, \mathrm{~d} / \mathrm{h}=0.01$, and $k_{0} h=4$.

is important for breaking ice covers. But it may not be desirable for manmade structures. It can be observed that for simply supported or built-in structures, the strain deviation is not much except near the edge. In this case, the effect is very much localized.

In Fig. 8, the plate deflection $\operatorname{Re}\left(\eta / H_{0}\right)$ is plotted versus $x / h$ for various values of $E I / \rho g h^{4}$ for a free-edge plate. It is observed that around the edge, there exists a decaying progressive wave and infinite number of evanescent wave modes apart from the flexural gravity wave. Away from the edge, all the energy associated with the decaying progressive wave and the infinite sum of evanescent wave modes has been attenuated sufficiently and they offer negligible contribution compared to the progressive wave. In other words, both the decaying progressive wave and evanescent wave modes are forms of localized energy. Large wave transmission always leads to a large plate deflection. From the figure, it is observed that the plate deflection is less for higher values of the flexural rigidity $E I / \rho g h^{4}$, as a rigid plate does not deform very much. There is also a phase shift of the wave elevation for the change in flexural rigidity $E I / \rho g h^{4}$ of the plate.

Figures 9 and 10 show the variation of the real and imaginary parts of plate deflection $\eta / H_{0}$ versus $x / h$ for different kinds of edge conditions. The asymptotic trend of solutions (12a) and (12b) is a progressive wave, with the real and imaginary components having the same magnitude and shifted in phase by a quarter-wavelength, as shown in Figs. 9 and 10. For a free-edge plate, $\eta / H_{0}$ is maximum at the edge and then attends a periodic steady state as the distance from the edge increases, while for a simply supported plate or a built-in edge plate, $\eta / H_{0}$ increases from zero at the edge to a periodic steady state as the waves progress below the plate. The values of $\eta / H_{0}$ for different edge conditions are similar away from the edge. In general, the plate deflection is maximum for the free edge and minimum for the built-in edge. There is also a phase shift as the edge condition changes.

\section{CONCLUSIONS}

The hydroelastic interaction of surface waves with a semi-infinite horizontal elastic plate floating on the free sur- 
face is analyzed using the eigenfunction expansions method and the orthogonality property of eigenfunctions in the platecovered region. The hydrodynamic characteristics are compared for plates having three different types of edge conditions. The three types of edges considered in the present study are (i) free edge, (ii) simply supported edge, and (iii) built-in edge. It is observed that the hydrodynamic behaviors depend on the wave conditions, the geometrical settings and the edge conditions. The built-in edge condition induces the maximum wave reflection and the minimum wave transmission. The free edge condition leads to the maximum plate deflection.

\section{ACKNOWLEDGMENTS}

This research was sponsored by the Hong Kong Research Grants Council under Grants No. HKU 7068/00E and No. NSFC/HKU 8. T.L.Y. was supported financially by a Post-Doctoral Fellowship for the Area of Excellence in Harbour and Coastal Environment Studies.

${ }^{1}$ H. Kagemoto, F. Masataka, and M. Motohiko, "Theoretical and experimental predictions of the hydroelastic response of a very large floating structure in waves," Appl. Ocean Res. 20, 135 (1998).

${ }^{2}$ R. E. D. Bishop and W. G. Price, Hydroelasticity of Ships (Cambridge University Press, London, 1979).

${ }^{3}$ S. Gran, A Course in Ocean Engineering (Elsevier, London, 1992).

${ }^{4} \mathrm{M}$. Kashiwagi, "Research on hydroelastic responses of VLFS: Recent progress and future work," Intl. J. Offshore and Polar Engng. 10, 81 (2000)

${ }^{5}$ C. Fox and V. A. Squire, "On the oblique reflection and transmission of ocean waves at shore fast sea ice," Philos. Trans. R. Soc. London, Ser. A 347, 185 (1994).

${ }^{6}$ V. A. Squire, J. P. Dugan, P. Wadhams, P. J. Rottier, and A. K. Liu, “Ocean waves and sea ice," Annu. Rev. Fluid Mech. 27, 115 (1995).

${ }^{7} \mathrm{M}$. H. Meylan, "The wave response of floating thin plates of shallow draft by a variational method," Proceedings of the 6th International Conference on Offshore and Polar Engineering 3, 328 (1996).

${ }^{8} \mathrm{M}$. H. Meylan, "The forced vibration of a thin plate floating on an infinite liquid," J. Sound Vib. 205, 581 (1997).

${ }^{9}$ N. J. Balmforth and R. V. Craster, "Ocean waves and ice sheets," J. Fluid Mech. 395, 89 (1999).

${ }^{10} \mathrm{M}$. Ohkusu and Y. Nanba, "Analysis of hydroelastic behavior of a large floating platform of thin plate configurations in waves," Proceedings of the International Workshop on Very Large Floating Structures, Hayama, Japan (1996), p. 143.

${ }^{11}$ I. V. Sturova, "The oblique incidence of surface waves onto the elastic band," Proceedings of the 2nd International Conference on Hydroelasticity in Marine Technology, Fukuoka, Japan (1998), p. 239.

${ }^{12}$ J. W. Kim and R. C. Ertekin, "An eigenfunction-expansion method for predicting hydroelastic behavior of a shallow-draft VLFS," Proceedings of the 2nd International Conference on Hydroelasticity in Marine Technology, Fukuoka, Japan (1998), p. 47.

${ }^{13}$ Y. Nanba and M. Ohkusu, "Hydroelastic behavior of artificial islands in waves," International J. Offshore and Polar Engng. 9, 39 (1999).

${ }^{14}$ J. N. Newman, "Wave effects on deformable bodies," Appl. Ocean Res. 16, 47 (1994)

${ }^{15} \mathrm{C}$. Wu, E. Watanabe, and T. Utsunomiya, "An eigenfunction expansion matching method for analyzing the wave-induced responses of an elastic floating plate," Appl. Ocean Res. 17, 301 (1995).

${ }^{16}$ T. H. Havelock, "Forced surface waves on water," Philos. Mag. 8, 569 (1929).

${ }^{17} \mathrm{P}$. F. Rhodes-Robinson, "On surface waves in the presence of immersed vertical boundaries I,” Q. J. Mech. Appl. Math. 32, 109 (1979).

${ }^{18}$ P. F. Rhodes-Robinson, "On surface waves in the presence of immersed vertical boundaries II," Q. J. Mech. Appl. Math. 32, 125 (1979).

${ }^{19}$ A. Chakrabarti and T. Sahoo, "The effect of surface tension in porous wavemaker problems," J. Aust. Math. Soc. B, Appl. Math. 39, 539 (1998).

${ }^{20} \mathrm{~S}$. Timoshenko and S. Woinowsky-Krieger, Theory of Plates and Shells, 2nd ed. (McGraw-Hill, Singapore, 1970).

${ }^{21} \mathrm{P}$. Wadhams, "The seasonal ice zone," in The Geophysics of Sea Ice, edited by N. Untersteiner, NATO ASI Series; Series B, Physics (Plenum, New York, 1986), Vol. 146, pp. 825-991.

${ }^{22}$ C. Fox and V. A. Squire, "Reflection and transmission characteristics at the edge of shore fast sea ice," J. Geophys. Res., [Oceans] 95, 11629 (1990). 